\title{
The tuberculocidal activity of polyaniline and functionalised polyanilines
}

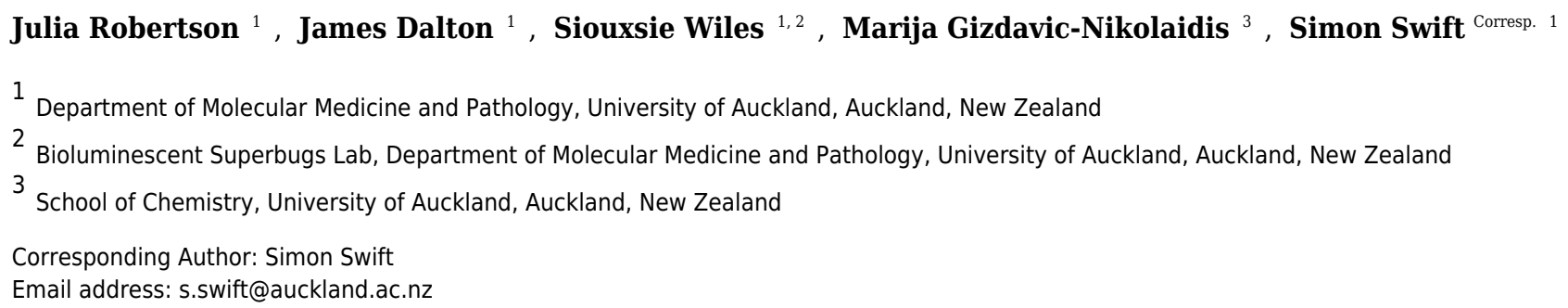

Tuberculosis is considered a leading cause of death worldwide. More than $95 \%$ of cases and deaths occur in low- and middle-income countries. In resource-limited countries, hospitals often lack adequate facilities to manage and isolate patients with infectious tuberculosis (TB), relying instead on personal protective equipment, such as facemasks, to reduce nosocomial transmission of the disease. Facemasks impregnated with an antimicrobial agent may be a cost-effective way of adding an extra level of protection against the spread of TB by reducing the risk of disease transmission. Conducting polymers, such as polyaniline (PANI), and their functionalised derivatives are a novel class of antimicrobial agents with potential as non-leaching additives to provide contamination resistant surfaces. We have investigated the antimicrobial action of PANI and a functionalised derivative, poly-3-aminobenzoic acid (P3ABA), against mycobacteria and have determined the optimal treatment time and concentration to achieve significant knockdown of Mycobacterium smegmatis and Mycobacterium tuberculosis on an agar surface. Results indicated that P3ABA is a potential candidate for use as an antituberculoid agent in facemasks to reduce TB transmission. 
3 Julia Robertson $^{1}$, James Dalton ${ }^{1}$, Siouxsie Wiles ${ }^{1}$, Marija Gizdavic-Nikolaidis ${ }^{2}$ and Simon Swift ${ }^{1}$

$4{ }^{1}$ Department of Molecular Medicine and Pathology, and ${ }^{2}$ School of Chemical Sciences, University of Auckland, Auckland, New Zealand

6 Corresponding Author: Simon Swift, Department of Molecular Medicine and Pathology, Faculty

7 of Medical and Health Sciences, University of Auckland, Private Bag 92019, Auckland, New

8 Zealand. Ph +64 93737599 ext 86273. Email s.swift@auckland.ac.nz.

\section{Abstract}


10 Tuberculosis is considered a leading cause of death worldwide. More than $95 \%$ of cases and

11 deaths occur in low- and middle-income countries. In resource-limited countries, hospitals often

12 lack adequate facilities to manage and isolate patients with infectious tuberculosis (TB), relying

13 instead on personal protective equipment, such as facemasks, to reduce nosocomial transmission

14 of the disease. Facemasks impregnated with an antimicrobial agent may be a cost-effective way

15 of adding an extra level of protection against the spread of TB by reducing the risk of disease

16 transmission. Conducting polymers, such as polyaniline (PANI), and their functionalised

17 derivatives are a novel class of antimicrobial agents with potential as non-leaching additives to

18 provide contamination resistant surfaces. We have investigated the antimicrobial action of PANI

19 and a functionalised derivative, poly-3-aminobenzoic acid (P3ABA), against mycobacteria and

20 have determined the optimal treatment time and concentration to achieve significant knockdown

21 of Mycobacterium smegmatis and Mycobacterium tuberculosis on an agar surface. Results

22 indicated that P3ABA is a potential candidate for use as an anti-tuberculoid agent in facemasks to

23 reduce TB transmission.

\section{Introduction}


25 Tuberculosis (TB) ranks alongside the human immunodeficiency virus (HIV) as a leading cause

26 of death worldwide (1). It has been estimated that in 20149.6 million people contracted, and 1.5

27 million people died from, TB (1). While the global burden of TB has been decreasing over the

28 last decade, more needs to be done as most deaths from TB are preventable and infections due to

29 drug resistant TB are on the rise (1). More than 95\% of cases and deaths occur in low- and

30 middle-income countries (LMICs), notably in South-East Asia and African regions (1). Multidrug

31 resistant tuberculosis (MDR-TB), caused by Mycobacterium tuberculosis that is resistant to both

32 isoniazid and rifampin, is a threat to TB control $(1,2)$. It is estimated that 480,000 people $(5 \%$ of

33 cases worldwide) developed MDR-TB in 2014 while an estimated 190,000 people (13\% of

34 deaths worldwide) died from MDR-TB infection (1). MDR-TB is difficult to cure as treatment is

35 required for longer periods of time with more costly drugs with toxic side effects (1).

36 Conducting polymers (CPs) and their functionalised derivatives are a novel class of antimicrobial

37 agents that may be used as an additive to provide contamination resistant surfaces. PANI and its

38 functionalised derivatives (fPANIs) comprise a widely studied class of conducting polymers (3).

39 Utilisation of PANI for potential applications is restricted because of its insolubility in common

40 solvents, which renders it difficult to process $(4,5)$. fPANIs are easily and inexpensively

41 synthesised using substituted aniline monomers, which improves the solubility, and thus

42 processability, of the resulting polymer $(4,5)$. PANI and fPANIs have good thermal stability and

43 have been incorporated as melt blends in plastics (6) and electrospun in nanofibers (7) where

44 antimicrobial activity is retained. These novel antimicrobial agents have broad spectrum activity

45 against gram-negative and gram-positive bacteria, but activity against mycobacteria has not been

46 reported $(8,9)$. Preliminary studies have suggested fPANIs have a multifunctional mechanism of

47 action that act, in part, via perturbation of aerobic energy metabolism to cause lethal oxidative

48 damage (8). We hypothesise that there will be activity against mycobacteria given that they are 
49 obligate aerobes and are sensitive to agents that interfere with aerobic energy metabolism (10-

50 12). Any role for conduction in antimicrobial activity is unclear, $\mathrm{P} 3 \mathrm{ABA}$ is $10^{3}-10^{4}$ less

51 conductive than PANI $(7,13)$.

52 In 2006, the WHO developed the 'Stop TB strategy' with the aim of diminishing the global TB

53 burden (1). One vital component of this strategy is improved infection control measures in health

54 facilities to reduce transmission, where effective use of personal protective equipment (PPE) is

55 necessary $(1,2,14,15)$. However, healthcare facilities in LMICs rarely implement recommended

56 TB infection control measures due to financial and resource constraints $(2,16,15,17)$ and health

57 care workers (HCWs) are at greater risk of contracting drug-resistant TB as a result $(18,19)$.

58 Facemasks fabricated from materials impregnated with an antimicrobial agent are a potential cost

59 effective strategy to protect against the spread of TB (20). Antimicrobial facemasks may decrease

60 the risk of TB transmission that occurs due to reuse of contaminated facemasks or reuse of

61 facemasks that have been structurally compromised during decontamination $(2,20,21)$.

62 Contamination resistant facemasks are a realistic infection control measure for resource-

63 constrained settings as they would not need to be replaced as frequently as disposable masks.

64 This would reduce the overall expense of providing facemasks to HCWs and could increase

65 facemask availability.

66 In this study we have investigated the antimicrobial action of PANI and an PPANI, P3ABA,

67 against mycobacteria genetically tagged for bioluminescence as a marker for cellular viability.

68 Bioluminescence is the production of light via a luciferase catalysed reaction (22). As tagged

69 cells only produce a signal when alive, bioluminescence is an excellent reporter to rapidly assay

70 for antimicrobial compounds, non-destructively and in real-time, in microtitre plate formats using

71 a luminometer, or in vivo using sensitive imaging equipment $(23,24)$. We determined the optimal

72 treatment time and concentration to achieve a $>10^{3}$-fold knockdown for bioluminescently-tagged 
73 strains of M. smegmatis and M. tuberculosis inoculated onto a solid surface. Results indicated

74 that $\mathrm{P} 3 \mathrm{ABA}$ is a potential candidate for use as an antimicrobial agent incorporated into materials

75 and PPE in the patient environment to reduce TB transmission.

\section{Methods and materials}

77 Bacterial strains and growth conditions. M. smegmatis ATCC 700084 was tagged with an

78 integrating plasmid (pMVhspLuxABG13CDE) containing the bacterial luciferase (lux) operon

79 and designated BSG200 using a standard method $(25,26)$. M. tuberculosis reference strain ATCC

8027294 and $M$. tuberculosis clinical isolate Rangipo N were similarly tagged and designated

81 BSG001 and BSG002, respectively $(27,28)$. All strains were grown at $37^{\circ} \mathrm{C}$, with $200 \mathrm{rpm}$

82 agitation where appropriate. The University of Auckland Institutional Biological Safety

83 Committee approved the construction and use of genetically modified risk group 2 mycobacteria

84 (GMO11-UA007). The New Zealand Environmental Protection Agency approved the

85 construction and use of genetically modified risk group 3 Mycobacterium tuberculosis

86 (APP201346).

87 Media and chemicals. All strains were cultured in Middlebrook 7H9 broth supplemented with

$88 \quad 10 \%$ Albumin Dextrose Catalase (ADC) and 0.5\% glycerol (subsequently referred to as

89 supplemented 7H9) or on Middlebrook 7H11 agar supplemented with 10\% Oleic Albumin

90 Dextrose Catalase (OADC) and 5\% glycerol (subsequently referred to as supplemented 7H11).

91 Bacterial growth media were obtained from Fort Richard, Auckland. For the starting inocula,

$92 \quad 0.05 \%$ Tween 80 (Sigma Aldrich) was added to the supplemented $7 \mathrm{H} 9$ to avoid cellular

93 agglomeration. To generate the experimental inocula, cells were diluted in supplemented 7H9

94 without tween 80. PANI and P3ABA were synthesised via chemical oxidation of aniline and 3-

95 aminobenzoic acid monomers, respectively (8). 
96 Antibacterial action of surface incorporated PANI and P3ABA against $M$. smegmatis.

97 Aliquots $(200 \mu \mathrm{l})$ of supplemented $7 \mathrm{H} 11$ agar containing 8\% PANI, 10\% PANI, 1\% P3ABA, 2\%

98 P3ABA or 3.5\% P3ABA were added to wells of a black 96-well plate (Perkin Elmer) and allowed

99 to set. In each well the surface of the agar was inoculated with $\sim 10^{4}$ CFU M. smegmatis BSG200

100 in $10 \mu \mathrm{l}$ and incubated at $37^{\circ} \mathrm{C}$. At $15 \mathrm{~min}, 30 \mathrm{~min}$ and $120 \mathrm{~min}$ M. smegmatis BSG200 cells were

101 recovered by addition of $190 \mu \mathrm{l}$ supplemented 7H9 onto the agar, pipetting up and down 8-times,

102 and transfer of the broth to a fresh 96 -well plate. This plate was incubated at $37^{\circ} \mathrm{C}$ and

103 bioluminescence was measured using a $\operatorname{Victor}^{\mathrm{TM}} X$ light luminescence plate reader (2030-0010)

104 (Perkin Elmer) at $0 \mathrm{~h}$ and $24 \mathrm{~h}$ incubation. Uninoculated wells were measured to generate

105 background luminescence readings. The experiment was performed in triplicate using

106 independent cultures.

107 Antibacterial action of surface incorporated PANI and P3ABA against M. tuberculosis. The

108 method developed for M. smegmatis was adapted to examine the tuberculocidal action of surface

109 incorporated PANI and P3ABA. M. tuberculosis BSG001 or M. tuberculosis BSG002 were

110 inoculated onto agar in black 96-well plates and rescued at the stated time points. The recovered

111 cells were incubated at $37^{\circ} \mathrm{C}$. The level of bioluminescence was measured after approximately 21

112 days using a Victor ${ }^{\mathrm{TM}} X$ light luminescence plate reader and growth was assessed visually. The

113 experiments were done in triplicate using independent cultures.

114 Statistical analyses. Statistical analysis was performed using GraphPad Prism software version 6

115 made (GraphPad Software, Inc.). Analysis of the effect of PANI and P3ABA treatment was based

116 on the bioluminescence measurement $24 \mathrm{~h}$ post-rescue for $M$. smegmatis and 21 days post-rescue

117 for M. tuberculosis. The Friedman test was used to detect differences in antimicrobial treatments

118 utilising a 5\% level of statistical significance. To determine which PANI or P3ABA concentration 
119 could achieve significant knockdown with the shortest exposure time, where 15, 30 and 120

120 minute challenges were made, Dunn's multiple comparison post-hoc test was used.

\section{Results}

\section{Antibacterial action of surface incorporated PANI and P3ABA against M. smegmatis}

123 BSG200. To investigate the activity of PANI and P3ABA against $M$. tuberculosis, the agar

124 surface decontamination assay was first optimised using M. smegmatis BSG200. M. smegmatis is

125 used as a safer and faster growing surrogate for the highly pathogenic M. tuberculosis (29). The

126 surface of agar with $10 \%$ and $8 \%$ PANI reduced bioluminescence measurements from $M$.

127 smegmatis BSG200 to background levels after $30 \mathrm{~min}$ and $120 \mathrm{~min}$ exposure times, respectively

128 (Fig. 1A). The activity of PANI was statistically significant (Friedman test, P value: 0.0033), with

129 both $10 \%$ and $8 \%$ PANI treatment differing significantly from untreated cells at the 120 min time

130 point (Dunn's multiple comparison post-hoc test).

131 Agar containing 2\% P3ABA and 3.5\% P3ABA reduced bioluminescence levels from surface-

132 inoculated M. smegmatis BSG200 cells to that of uninoculated agar following a 15 min treatment

133 (Fig. 1B). Agar containing 1\% P3ABA demonstrated variable activity with consistent knockdown

134 of surface-inoculated M. smegmatis BSG200 cells occurring after 30 min exposure (Fig. 1B). The

135 overall antimicrobial action of 3.5\% P3ABA was statistically significant (Friedman test, P value:

136 0.0044). Dunn's multiple comparison test confirmed the significant activity of 3.5\% P3ABA in a

$13715 \mathrm{~min}$ treatment time. Having established the protocol for fast-growing, non-pathogenic

138 mycobacteria, testing was extended to the slow-growing human pathogen M. tuberculosis.

139 Antibacterial action of surface incorporated PANI and P3ABA against M. tuberculosis

140 BSG001. The activity of the polymers against $M$. tuberculosis was first investigated with the

141 tagged laboratory reference strain M. tuberculosis BSG001. Both 10\% and 8\% PANI agar did not 
142 mediate a reduction of $M$. tuberculosis BSG001 after treatment for 120 min (Fig. 2A). This is in

143 contrast to the action of PANI against M. smegmatis BSG200 (Fig. 1A). A 15 min exposure of

144 surface-inoculated M. tuberculosis BSG001 cells to $\%$ and 3.5\% P3ABA agar reduced

145 bioluminescence levels to that of background levels (Fig. 2B). The action of 1\% P3ABA agar

146 against surface-inoculated $M$. tuberculosis BSG001 was variable with most cells being killed

147 after 120 min treatment (Fig. 2B). Knockdown of M. tuberculosis BSG001 following $30 \mathrm{~min}$

148 exposure to the agar surface containing 3.5\% P3ABA was statistically significant (Friedman test,

149 P value: 0.0015; Dunn's multiple comparison post-hoc test).

150 Antibacterial action of surface incorporated PANI and P3ABA against the clinical isolate

151 M. tuberculosis BSG002. M. tuberculosis BSG002 was tested using the surface decontamination

152 assay to ascertain the activity of PANI and P3ABA against a clinical isolate. Similar to the

153 activity against $M$. tuberculosis BSG001, both 10\% and 8\% PANI incorporated into agar did not

154 mediate any meaningful knockdown of M. tuberculosis BSG002 (Fig. 3A).

155 As for M. smegmatis BSG200 and M. tuberculosis BSG001, 2\% and 3.5\% P3ABA agar reduced

156 bioluminescence levels from M. tuberculosis BSG002 to that of the uninoculated agar (Fig. 3B).

157 Surface incorporated 1\% P3ABA knocked M. tuberculosis BSG002 cells down to slightly above 158 background levels after a 30 min treatment (Fig. 3B). Treatment of M. tuberculosis BSG002 with

159 surface incorporated 3.5\% P3ABA was statistically significant (Friedman test, P value: 0.0042).

160 Dunn's multiple comparison test confirmed the significant activity of 3.5\% P3ABA in a 15 min

161 treatment time.

\section{Discussion}

163 PANI and P3ABA have previously been shown to have broad spectrum activity against a range of

164 gram-positive and gram-negative bacteria $(8,9)$. The results presented here demonstrate the 
165 previously untested activity of these compounds against mycobacteria. Only a 15 min exposure to

$1662 \%$ P3ABA was required to mediate knockdown of M. smegmatis and M. tuberculosis. This time

167 interval was the shortest that could be tested due to the time constraints involved in the local

168 Standard Operating Procedures for M. tuberculosis laboratory work. It is possible that P3ABA

169 can decontaminate mycobacteria laden surfaces in less than a 15 min exposure.

170 The WHO recommends that HCWs in high incidence TB areas wear respirators to protect against

171 TB transmission (1). More than 50\% of new MDR-TB cases occur within hospitals and

172 communities among people that haven't been previously treated for TB, which highlights the lack

173 of adequate infection control measures in high MDR-TB incidence areas $(2,14,17)$. Improved

174 TB infection control is essential to curtail the development and spread of drug resistance (2).

175 There are not many studies that address the efficacy of infection control measures on reducing

176 transmission; however, there is limited evidence which suggests that the incidence of TB

177 infection decreases after execution of control measures (19).

178 Respirators are intended to protect the wearer from inhaling infectious particles by filtering small

179 infectious droplets $(30,31)$. This type of PPE is effective at preventing inhalation of infectious

180 particles; however, the cost of the product renders respirators unobtainable for healthcare

181 facilities in LMICs, which decreases the level of protection offered $(2,30)$. Efforts to curtail the

182 spread of MDR-TB is hampered by insufficient funding (1). The funding gaps associated with a

183 full response to the global TB epidemic in LMICs has been steadily increasing, amounting to

184 US\$ 1.4 billion in 2015 (1).

185 Facemasks are worn over nose and mouth to establish a barrier between the respiratory tract and

186 splashes and droplets in the external environment $(30,31)$. Facemasks offer less protection

187 against $M$. tuberculosis transmission as they have a lower filtration efficiency and a higher degree

188 of face seal leakage than respirators (30-32). In a resource-limited setting where there are few/no 
189 respirators available, wearing of facemasks may help decrease transmission even if each

190 individual HCW is not completely protected $(2,32,33)$. Use of a facemask that reduces

191 inhalation of particles by $50 \%$ has been estimated to give the same level of protection as a

192 doubling of room ventilation, but at a much lower cost (2). The greater affordability of facemasks

193 is associated with fewer availability issues than respirators (2).

194 Facemasks provide important protection in resource-limited settings where recommended

195 infection control measures are not feasible. However, healthcare facilitates in these areas are not

196 able to reliably provide HCWs with sterile facemasks, increasing the risk of TB transmission in

197 high bacterial load situations, such as an infectious patient in a poorly ventilated room (34-36).

198 Used contaminated facemasks can be a source of infection either due to penetration of particles

199 through the facemask into the respiratory tract of the wearer or release of aerosols back into the

200 air $(34,35)$. Used facemasks isolated from a hospital have been shown to have bacterial

201 contamination on the outside of the mask (34). M. abscessus, used as a surrogate for $M$.

202 tuberculosis, is able to survive on respiratory PPE for 5 days and can be transferred to gloves

203 during handling (35). Decontamination of facemasks by HCWs may also cause reaerosolisation

204 of TB bacilli (36). A facemask impregnated with an antimicrobial agent would ameliorate the risk

205 of TB transmission associated with these non-recommended practises. Bacteria that come into

206 contact with the facemask surface would be killed, facilitating safer reuse and disposal as well as

207 eliminating the need to decontaminate (37).

208 There are several commercially available decontaminating facemasks; however, there is limited

209 published work on the efficacy of these facemasks. Two notable examples include silver-based

210 facemasks and quaternary ammonium compound (QAC) based facemasks $(38,39)$. The cell wall

211 of M. tuberculosis is protective against penetration of silver nanoparticles into the cytoplasm as

212 disruption with chloroform was required for inhibitory activity (40). Mycobacterium avium, a 
213 pathogenic mycobacterium, was reduced slightly in number after a 24-48 h treatment with silver

214 nanoparticles, which suggests that the bactericidal activity of silver nanoparticles against $M$.

215 tuberculosis in a relevant time frame (15 min) would be negligible $(41,42)$. Furthermore, the

216 potential for development of resistance to silver and cross-resistance to antibiotics reduces the

217 suitability of silver as an antimycobacterial agent. Following a single exposure to silver

218 nanoparticles, $M$. smegmatis developed resistance to silver nanoparticles, silver nitrate and the

219 antibiotic isoniazid (43). QACs have been demonstrated to be active against hydrophilic bacteria

220 that have a negatively charged cell surface, including LPS-expressing gram-negative bacteria and

221 teichoic-acid containing gram-positive bacteria; however, the efficacy against hydrophobic

222 mycobacteria is limited $(44,45)$.

223 Our findings presented here identify P3ABA as a suitable candidate for the production of

224 antimicrobial surfaces in the patient environment, including facemask materials due to the low

225 cost of synthesis, environmental and thermal stability, and lack of mammalian cytotoxicity $(3,4)$.

226 The broad spectrum activity of fPANI may confer protection against other respiratory pathogens

227 that also have a high burden in LMICs, such as bacterially derived pneumonia (32).

228 Challenges remain for researchers; the first is to demonstrate tuberculocidal activity of P3ABA in

229 facemask material. We believe this is likely as melt blends of PANI in low density polyethylene

230 (6) and P3ABA electrospun in nanofibers of poly(lactic acid) $(4,13)$ retain antimicrobial activity.

231 Assuming an effective facemask can be fabricated it will be important to establish that it is

232 effective in reducing TB transmission and to determine the expected usage lifetime to give

233 guidelines as to when to discard a used mask. As users may be tempted to reuse masks,

234 irrespective of mask age, for the same reasons that they currently reuse infected masks; thought

235 will still need to be given to removing the reasons for extended use and to enforcing expiration 236 guidelines. 


\section{Conclusion}

238 A surface self-decontamination assay was established and used to test the activity of two novel

239 antimicrobial agents, PANI and P3ABA, against mycobacteria. PANI is active against $M$.

240 smegmatis BSG200 after 120 min exposure; however, it did not mediate surface self-sanitisation

241 of M. tuberculosis BSG001 or M. tuberculosis BSG002. A 15 min exposure to a surface

242 containing 3.5\% P3ABA was sufficient to kill all three strains tested. Therefore, P3ABA has

243 potential to be used to create anti-tubercular facemasks, which would serve as a cost-effective TB

244 infection control measure in low-resource, high TB burden areas.

245 Acknowledgments

246 The authors thank Sudip Ray, Adeline Le Cocq, Chris Wilcox and Walt Wheelwright for purified

247 PANI and P3ABA.

248 References

249 1. WHO. 2015. Global tuberculosis report 2015 (WHO/HTM/TB/2015.22).

250 2. Menon S. 2013. Preventing nosocomial MDR TB transmission in sub Saharan Africa:

251 where are we at? Glob J Health Sci 5:200-10.

252 3. Dhand C, Das M, Datta M, Malhotra BD. 2011. Recent advances in polyaniline based

253 biosensors. Biosens Bioelectron 26:2811-21.

254 4. Gizdavic-Nikolaidis MR, Ray S, Bennett JR, Swift S, Bowmaker GA, Easteal AJ.

255 2011. Electrospun poly(aniline-co-ethyl 3-aminobenzoate)/poly(lactic acid) nanofibers and

256 their potential in biomedical applications. J Polym Sci Part A Polym Chem 49:4902-4910.

257 5. Pandey S, Annapoorni S, Malhotra BD. 1993. Synthesis and Characterization of 

26:3190-3193.

6. Nand A V., Swift S, Uy B, Kilmartin PA. 2013. Evaluation of antioxidant and antimicrobial properties of biocompatible low density polyethylene/polyaniline blends. J Food Eng 116:422-429.

7. Gizdavic-Nikolaidis MR, Ray S, Bennett JR, Easteal AJ, Cooney RP. 2010.

264 Electrospun functionalized polyaniline copolymer-based nanofibers with potential 265 application in tissue engineering. Macromol Biosci 10:1424-31.

266 8. Gizdavic-Nikolaidis MR, Bennett JR, Swift S, Easteal AJ, Ambrose M. 2011. Broad 267 spectrum antimicrobial activity of functionalized polyanilines. Acta Biomater 7:4204-9.

268 9. Shi N, Guo X, Jing H, Gong J, Sun C, Yang K. 2006. Antibacterial effect of the 269 conducting polyaniline. J Mater Sci Technol 22:289-290. 2015. Bactericidal mode of action of bedaquiline. J Antimicrob Chemother 1-10.

11. Leibert E, Danckers M, Rom WN. 2014. New drugs to treat multidrug-resistant tuberculosis: the case for bedaquiline. Ther Clin Risk Manag 10:597-602.

12. Koul A, Vranckx L, Dhar N, Göhlmann HWH, Özdemir E, Neefs J-M, Schulz M, Lu Mycobacterium tuberculosis to bedaquiline involves remodelling of bacterial metabolism. Nat Commun 5:3369. 
280 Copolymers. J Polym Sci Part A Polym Chem 48:1339-1347.

281 14. WHO. 2014. Drug-resistant TB Surveillance \& Response Supplement: Global tuberculosis 282 report $2014(\mathrm{WHO} / \mathrm{HQ} / \mathrm{TB} / 2014.12)$.

283 15. Buregyeya E, Nuwaha F, Verver S, Criel B, Colebunders R, Wanyenze R, Kalyango

284 JN, Katamba A, Mitchell EM. 2013. Implementation of tuberculosis infection control in 285 health facilities in Mukono and Wakiso districts, Uganda. BMC Infect Dis 13:360.

286 16. Gonzalez-Angulo Y, Geldenhuys H, Van As D, Buckerfield N, Shea J, Mahomed H,

287 Hanekom W, Hatherill M. 2013. Knowledge and acceptability of patient-specific

288 infection control measures for pulmonary tuberculosis. Am J Infect Control 41:717-722.

289 17. Kompala T, Shenoi S V., Friedland G. 2013. Transmission of tuberculosis in resource290 limited settings. Curr HIV/AIDS Rep 10:264-272.

291 18. O’Donnell MR, Jarand J, Loveday M, Padayatchi N, Zelnick J, Werner L, Naidoo K, 292 Master I, Osburn G, Kvasnovsky C, Shean K, Pai M, Van Der Walt M, Horsburgh CR, Dheda K. 2010. High Incidence of Hospital Admissions with Multidrug-Resistant and Extensively Drug-Resistant Tuberculosis among South African Health Care Workers. Ann Intern Med 153:516-522.

19. Joshi R, Reingold AL, Menzies D, Pai M. 2006. Tuberculosis among Health-Care Workers in Low- and Middle-Income Countries: A Systematic Review. PLoS Med 3:e494.

20. Yang P, Seale H, Macintyre CR, Zhang H, Zhang Z, Zhang Y, Wang X, Li X, Pang X, Wang Q. 2011. Mask-wearing and respiratory infection in healthcare workers in Beijing,

300 China. Braz J Infect Dis 15:102-108. 
H. 2014. Examining the policies and guidelines around the use of masks and respirators by healthcare workers in China, Pakistan and Vietnam. J Infect Prev 1-7.

304 305

22. Andreu N, Zelmer A, Wiles S. 2011. Noninvasive biophotonic imaging for studies of infectious disease. FEMS Microbiol Rev 35:360-394.

23. Andreu N, Zelmer A, Sampson SL, Ikeh M, Bancroft GJ, Schaible UE, Wiles S, Robertson BD. 2013. Rapid in vivo assessment of drug efficacy against Mycobacterium tuberculosis using an improved firefly luciferase. J Antimicrob Chemother 68:2118-27.

24. Andreu N, Fletcher T, Krishnan N, Wiles S, Robertson BD. 2012. Rapid measurement of antituberculosis drug activity in vitro and in macrophages using bioluminescence. $\mathrm{J}$ Antimicrob Chemother 67:404-14.

25. Wiles S, Ferguson K, Stefanidou M, Young DB, Robertson BD. 2005. Alternative luciferase for monitoring bacterial cells under adverse conditions. Appl Environ Microbiol 71:3427-3432.

26. Andreu N, Zelmer A, Fletcher T, Elkington PT, Ward TH, Ripoll J, Parish T, Bancroft GJ, Schaible U, Robertson BD, Wiles S. 2010. Optimisation of bioluminescent reporters for use with mycobacteria. PLoS One 5:e10777.

27. Colangeli R, Arcus VL, Cursons RT, Ruthe A, Karalus N, Coley K, Manning SD, Kim S, Marchiano E, Alland D. 2014. Whole genome sequencing of Mycobacterium tuberculosis reveals slow growth and low mutation rates during latent infections in humans. PLoS One 9:e91024.

28. Wang J, Pearce AN, Chan STS, Taylor RB, Page MJ, Valentin A, BourguetKondracki M-L, Dalton JP, Wiles S, Copp BR. 2016. Biologically Active Acetylenic 
Amino Alcohol and N-Hydroxylated 1,2,3,4-Tetrahydro- $\beta$-carboline Constituents of the New Zealand Ascidian Pseudodistoma opacum. J Nat Prod 79:607-10.

326

327

328

329

330

331

332

333

334

335

336

337

338

339

340

341

342

343

344

345

29. Chaturvedi V, Dwivedi N, Tripathi RP, Sinha S. 2007. Evaluation of Mycobacterium smegmatis as a possible surrogate screen for selecting molecules active against multi-drug resistant Mycobacterium tuberculosis. J Gen Appl Microbiol 53:333-337.

30. Coia JE, Ritchie L, Adisesh A, Makison Booth C, Bradley C, Bunyan D, Carson G, Fry C, Hoffman P, Jenkins D, Phin N, Taylor B, Nguyen-Van-Tam JS, Zuckerman M. 2013. Guidance on the use of respiratory and facial protection equipment. J Hosp Infect 85:170-182.

31. Cleveland JL, Robison VA, Panlilio AL. 2009. Tuberculosis Epidemiology, Diagnosis and Infection Control Recommendations for Dental Settings. J Am Dent Assoc 140:10921099.

32. MacIntyre CR, Wang Q, Rahman B, Seale H, Ridda I, Gao Z, Yang P, Shi W, Pang X, Zhang Y, Moa A, Dwyer DE. 2014. Efficacy of face masks and respirators in preventing upper respiratory tract bacterial colonization and co-infection in hospital healthcare workers. Prev Med (Baltim) 62:1-7.

33. Nicas M. 1995. Respiratory protection and the risk of Mycobacterium tuberculosis infection. Am J Ind Med 27:317-333.

34. Luksamijarulkul P, Aiempradit N, Vatanasomboon P. 2014. Microbial Contamination on Used Surgical Masks among Hospital Personnel and Microbial Air Quality in their Working Wards: A Hospital in Bangkok. Oman Med J 29:346-350.

35. Brosseau LM, McCullough NV, Vesley D. 1997. Bacterial survival on respirator filters 
346 and surgical masks. Jounal Am Biol Saf Assoc 2:32-43.

347 36. Rengasamy A, Zhuang Z, Berryann R. 2004. Respiratory protection against bioaerosols:

348 Literature review and research needs. Am J Infect Control 32:345-354.

349 37. Chellamani KP, Veerasubramanian D, Balaji RSV. 2013. Surgical Face Masks :

350 Manufacturing Methods and Classification 2:320-324.

351 38. Li Y, Leung P, Yao L, Song QW, Newton E. 2006. Antimicrobial effect of surgical 352 masks coated with nanoparticles. J Hosp Infect 62:58-63.

353 39. Tseng C-C, Pan Z-M, Chang C-H. 2016. Application of a quaternary ammonium agent

354 on surgical face masks before use for pre-decontamination of nosocomial infection-related

355 bioaerosols. Aerosol Sci Technol 50:199-210.

356 40. Praba VL, Kathirvel M, Vallayyachari K, Surendar K, Muthuraj M, Jesuraj PJ,

357 Govindarajan S, Raman K V. 2013. Bactericidal Effect of Silver Nanoparticles Against

$358<\mathrm{I}>$ Mycobacterium tuberculosis $</ \mathrm{I}>$. J Bionanoscience 7:282-287.

359 41. Islam MS, Larimer C, Ojha A, Nettleship I. 2013. Antimycobacterial efficacy of silver

360 nanoparticles as deposited on porous membrane filters. Mater Sci Eng C 33:4575-4581.

361 42. Miyamoto M, Yamaguchi Y, Sasatsu M. 2000. Disinfectant effects of hot water,

362 ultraviolet light, silver ions and chlorine on strains of Legionella and nontuberculous

363 mycobacteria. Microbios 101:7-13.

364 43. Larimer C, Islam MS, Ojha A, Nettleship I. 2014. Mutation of environmental

365 mycobacteria to resist silver nanoparticles also confers resistance to a common antibiotic.

366 BioMetals 27:695-702. 
367 44. Russell AD. 1996. Activity of biocides against mycobacteria. Soc Appl Bacteriol Symp $368 \quad$ Ser 25:87S-101S.

369 45. Gottenbos B, Van Der Mei HC, Klatter F, Nieuwenhuis P, Busscher HJ. 2002. In vitro 370 and in vivo antimicrobial activity of covalently coupled quaternary ammonium silane 371 coatings on silicone rubber. Biomaterials 23:1417-1423.

372 46. Shenoi S V., Escombe AR, Friedland G. 2010. Transmission of drug-susceptible and 373 drug-resistant tuberculosis and the critical importance of airborne infection control in the era of HIV infection and highly active antiretroviral therapy rollouts. Clin Infect Dis $\mathbf{5 0}$ Suppl 3:S231-7.

47. Dharmadhikari AS, Mphahlele M, Stoltz A, Venter K, Mathebula R, Masotla T, Lubbe W, Pagano M, First M, Jensen PA, Van Der Walt M, Nardell EA. 2012. Surgical face masks worn by patients with multidrug-resistant tuberculosis: Impact on infectivity of air on a hospital ward. Am J Respir Crit Care Med 185:1104-1109.

FIG 1 Antimicrobial action of surface incorporated PANI and P3ABA against M. smegmatis 
384 the surface of agar containing 3.5\% P3ABA (downward triangles), 2\% P3ABA (open circles) and

$3851 \%$ P3ABA (upward triangles). Following $15 \mathrm{~min}, 30 \mathrm{~min}$ and $120 \mathrm{~min}$ treatments, M. smegmatis

386 BSG200 cells were recovered by addition of supplemented $7 \mathrm{H} 9$ and incubation at $37^{\circ} \mathrm{C}$ in a fresh

387 96-well plate. The vertical axis represents the median bioluminescence measurements (given as

388 relative light units [RLU] per second) from recovered cells grown for $24 \mathrm{~h}$. The dashed line

389 denotes background luminescence of uninoculated wells.

390 FIG 2 Antimicrobial action of surface incorporated PANI and P3ABA against $M$. tuberculosis

391 BSG001. (A) M. tuberculosis BSG001 cells were exposed to the surface of agar containing $10 \%$

392 PANI (closed circles) and 8\% PANI (squares). (B) M. tuberculosis BSG001 cells were exposed to

393 the surface of agar containing 3.5\% P3ABA (downward triangles), 2\% P3ABA (open circles) and

$3941 \%$ P3ABA (upward triangles). Following $15 \mathrm{~min}, 30 \mathrm{~min}$ and $120 \mathrm{~min}$ treatments, $M$.

395 tuberculosis BSG001 cells were recovered by addition of supplemented 7H9 and incubation at

$39637^{\circ} \mathrm{C}$ in a fresh $96-w e l l$ plate. The vertical axis represents the median bioluminescence

397 measurements (given as relative light units [RLU] per second) from recovered cells grown for

398 approximately 21 days. The dashed line denotes background luminescence of uninoculated wells.

399 FIG 3 Antimicrobial action of surface incorporated PANI and P3ABA against $M$. tuberculosis

400 BSG002. (A) M. tuberculosis BSG002 cells were exposed to the surface of agar containing $10 \%$

401 PANI (closed circles) and 8\% PANI (squares). (B) M. tuberculosis BSG002 cells were exposed to

402 the surface of agar containing 3.5\% P3ABA (downward triangles), 2\% P3ABA (open circles) and

$4031 \%$ P3ABA (upward triangles). Following $15 \mathrm{~min}, 30 \mathrm{~min}$ and $120 \mathrm{~min}$ treatments, $M$.

404 tuberculosis BSG002 cells were recovered by addition of supplemented 7H9 and incubation at

$40537^{\circ} \mathrm{C}$ in a fresh $96-w e l l$ plate. The vertical axis represents the median bioluminescence

406 measurements (given as relative light units [RLU] per second) from recovered cells grown for

407 approximately 21 days. The dashed line denotes background luminescence of uninoculated wells. 

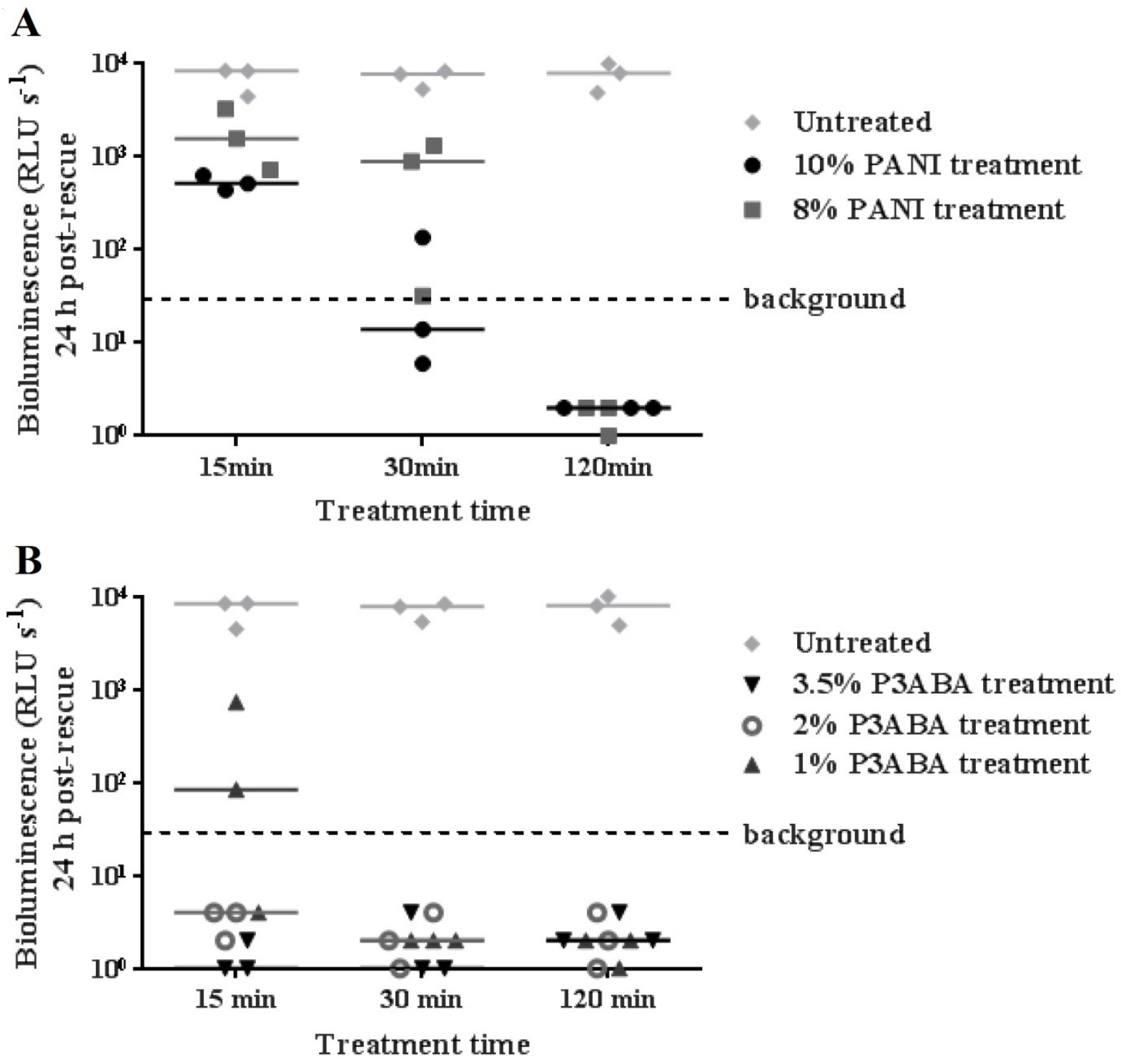

408 FIG 1 Antimicrobial action of surface incorporated PANI and P3ABA against M. smegmatis

409 BSG200. (A) M. smegmatis BSG200 cells were exposed to the surface of agar containing 10\%

410 PANI (closed circles) and 8\% PANI (squares). (B) M. smegmatis BSG200 cells were exposed to

411 the surface of agar containing 3.5\% P3ABA (downward triangles), 2\% P3ABA (open circles) and

$4121 \%$ P3ABA (upward triangles). Following $15 \mathrm{~min}, 30 \mathrm{~min}$ and $120 \mathrm{~min}$ treatments, M. smegmatis

413 BSG200 cells were recovered by addition of supplemented $7 \mathrm{H} 9$ and incubation at $37^{\circ} \mathrm{C}$ in a fresh 
414 96-well plate. The vertical axis represents the median bioluminescence measurements (given as 415 relative light units [RLU] per second) from recovered cells grown for $24 \mathrm{~h}$. The dashed line 416 denotes background luminescence of uninoculated wells. 

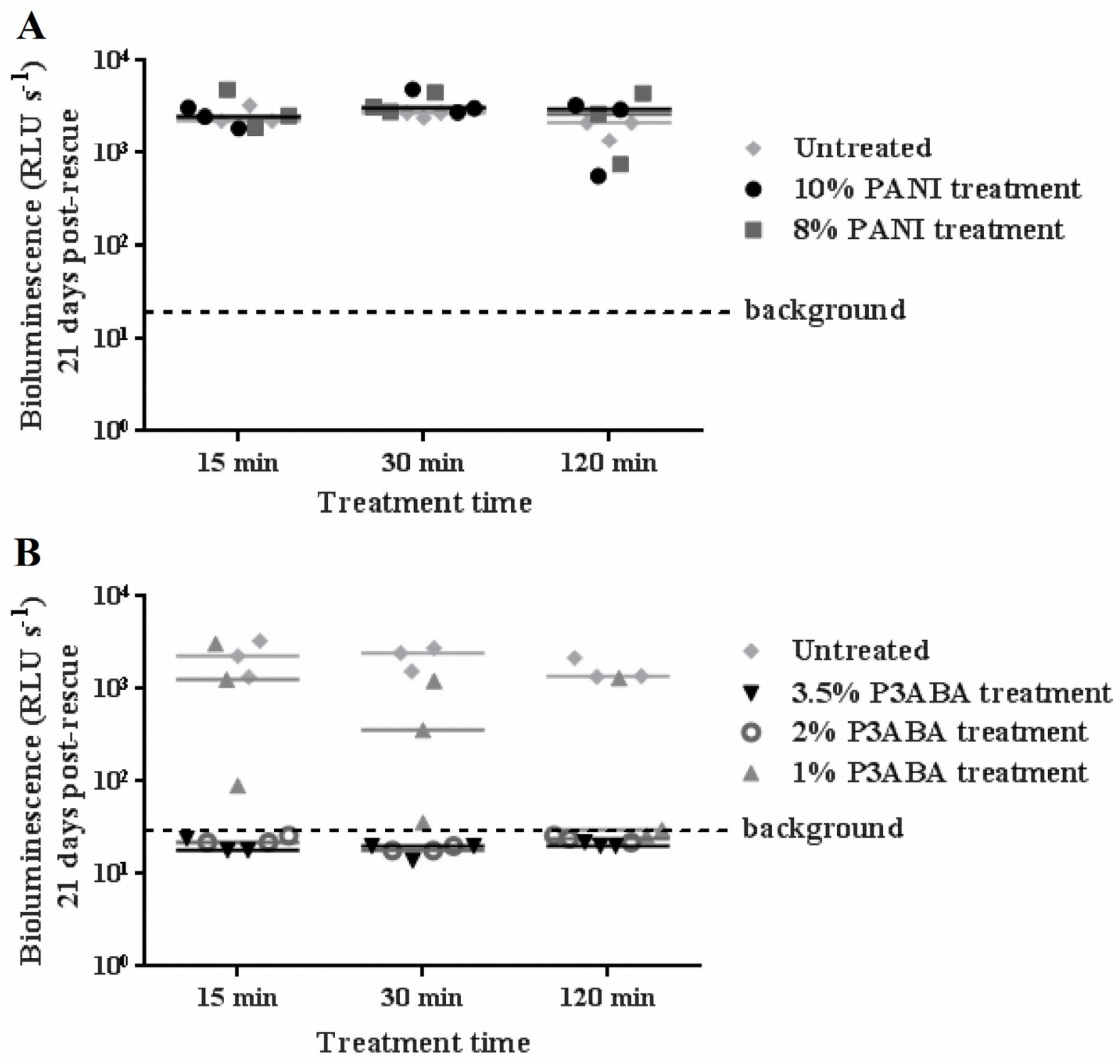

417 FIG 2 Antimicrobial action of surface incorporated PANI and P3ABA against $M$. tuberculosis

418 BSG001. (A) M. tuberculosis BSG001 cells were exposed to the surface of agar containing $10 \%$

419 PANI (closed circles) and 8\% PANI (squares). (B) M. tuberculosis BSG001 cells were exposed to

420 the surface of agar containing 3.5\% P3ABA (downward triangles), 2\% P3ABA (open circles) and

421 1\% P3ABA (upward triangles). Following $15 \mathrm{~min}, 30 \mathrm{~min}$ and $120 \mathrm{~min}$ treatments, $M$.

422 tuberculosis BSG001 cells were recovered by addition of supplemented 7H9 and incubation at 
$42337^{\circ} \mathrm{C}$ in a fresh $96-w e l l$ plate. The vertical axis represents the median bioluminescence

424 measurements (given as relative light units [RLU] per second) from recovered cells grown for

425 approximately 21 days. The dashed line denotes background luminescence of uninoculated wells. 

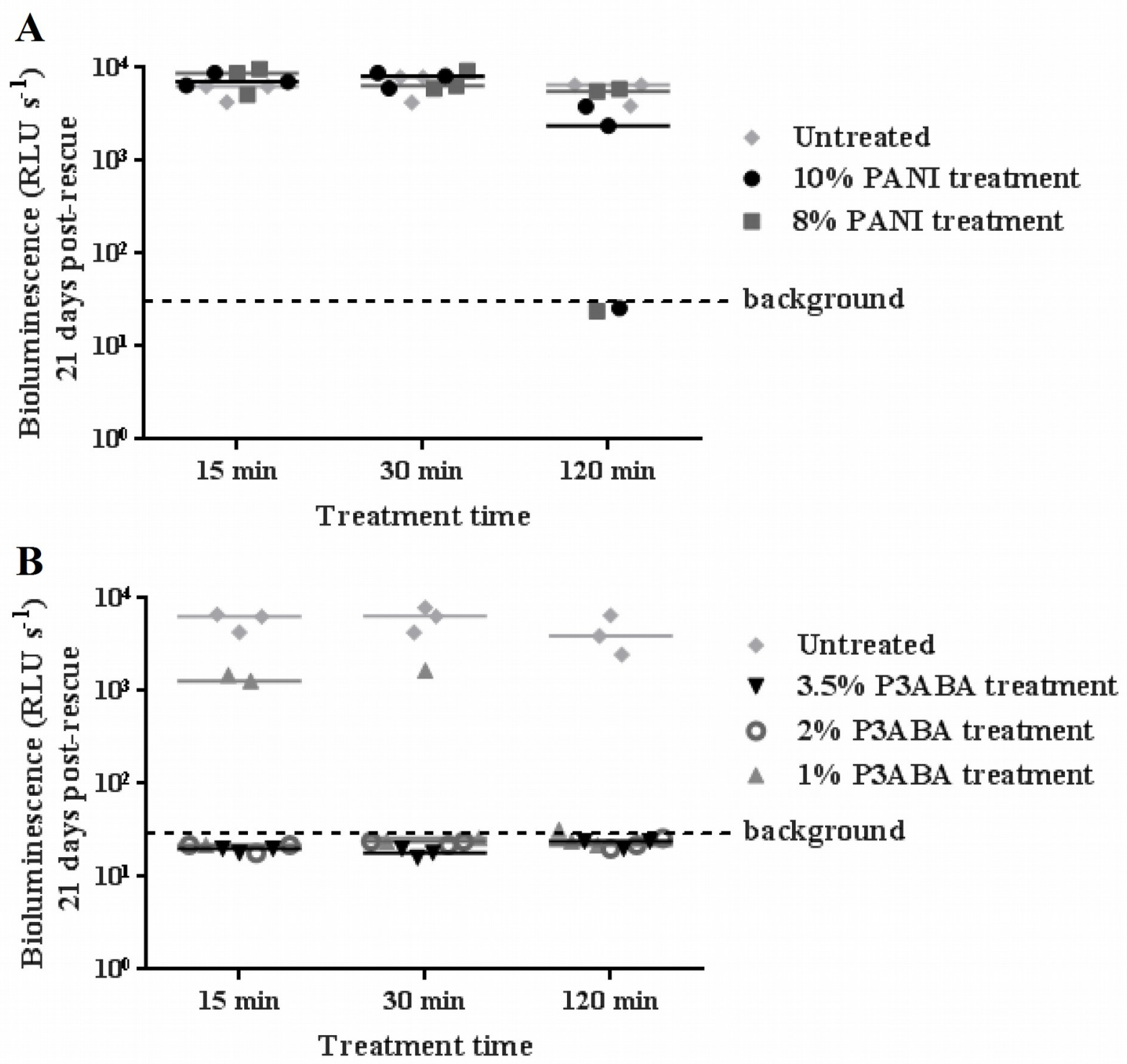

426 FIG 3 Antimicrobial action of surface incorporated PANI and P3ABA against $M$. tuberculosis

427 BSG002. (A) M. tuberculosis BSG002 cells were exposed to the surface of agar containing $10 \%$

428 PANI (closed circles) and 8\% PANI (squares). (B) M. tuberculosis BSG002 cells were exposed to 429 the surface of agar containing 3.5\% P3ABA (downward triangles), 2\% P3ABA (open circles) and $4301 \%$ P3ABA (upward triangles). Following $15 \mathrm{~min}, 30 \mathrm{~min}$ and $120 \mathrm{~min}$ treatments, $M$.

431 tuberculosis BSG002 cells were recovered by addition of supplemented 7H9 and incubation at 
$43237^{\circ} \mathrm{C}$ in a fresh 96 -well plate. The vertical axis represents the median bioluminescence 433 measurements (given as relative light units [RLU] per second) from recovered cells grown for

434 approximately 21 days. The dashed line denotes background luminescence of uninoculated wells. 